\title{
Extracellular matrix metabolism disorder induced by mechanical strain on human parametrial ligament fibroblasts
}

\author{
JIE MIN, BINGSHU LI, CHENG LIU, WENJUN GUO, SHASHA HONG, JIANMING TANG and LI HONG \\ Department of Gynecology and Obstetrics, Renmin Hospital of Wuhan University, Wuhan, Hubei 430060, P.R. China
}

Received January 18, 2016; Accepted February 6, 2017

DOI: $10.3892 / \mathrm{mmr} .2017 .6372$

\begin{abstract}
Pelvic organ prolapse (POP) is a global health problem that may seriously impact the quality of life of the sufferer. The present study aimed to investigate the potential mechanisms underlying alterations in extracellular matrix (ECM) metabolism in the pathogenesis of POP, by investigating the expression of ECM components in human parametrial ligament fibroblasts (hPLFs) subject to various mechanical strain loads. Fibroblasts derived from parametrial ligaments were cultured from patients with POP and without malignant tumors, who underwent vaginal hysterectomy surgery. Fibroblasts at generations 3-6 of exponential phase cells were selected, and a four-point bending device was used for $0,1,333$ or $5,333 \mu$ mechanical loading of cells at $0.5 \mathrm{~Hz}$ for $4 \mathrm{~h}$. mRNA and protein expression levels of collagen type I $\alpha 1$ chain (COL1A1), collagen type III $\alpha 1$ chain (COL3A1), elastin, matrix metalloproteinase (MMP) -2 and -9 , and transforming growth factor (TGF)- $\beta 1$ were detected by reverse transcription-quantitative polymerase chain reaction and western blotting, respectively. Under increased mechanical strain $(5,333 \mu)$, mRNA and protein expression levels of COL1A1, COL3A1 elastin and TGF- $\beta 1$ decreased, particularly COL1A1; however, mRNA and protein expression levels of MMP-2 and -9 were significantly increased, compared with the control group ( $0 \mu$ strain). Following $1,333 \mu$ mechanical strain, mRNA and protein expression levels of COL1A1, COL3A1 elastin and MMP-2 increased, and MMP-9 decreased, whereas no significant differences were observed in TGF- $\beta 1$ mRNA and protein expression levels. In conclusion, ECM alterations may be involved in pathogenesis of POP, with decreased synthesis and increased degradation of collagen and elastin. Furthermore, the TGF- $\beta 1$ signaling pathway may serve an important role in this process and thus may supply a new
\end{abstract}

Correspondence to: Professor Li Hong, Department of Gynaecology and Obstetrics, Renmin Hospital of Wuhan University, 238 Jiefang Road, Wuhan, Hubei 430060, P.R. China

E-mail: drhongli7777@gmail.com

Key words: mechanical strain, pelvic organ prolapse, extracellular matrix, matrix metalloproteinases, transforming growth factor- $\beta 1$ target and strategy for understanding the etiology and therapy of POP.

\section{Introduction}

Pelvic organ prolapse (POP) is a global health problem that may seriously impact the quality of life of the sufferer. It affects $\sim 50 \%$ of women $>50$ years and surgery is required in $20 \%$ of cases by the age of 80 (1). Vaginal childbirth, obesity and ageing are important risk factors for developing POP $(2,3)$ and occur when a loss of healthy attachment and support results in descent of the pelvic organs into the vaginal canal. The bladder, uterus and rectum are all located around the vaginal canal. Owing to the arrangement of these organs, bulging into the vaginal canal due to weakness of the supportive tissues for these organs is common. The descent of one or more out of the anterior vaginal wall, posterior vaginal wall, the uterus (cervix) or the apex of the vagina (vaginal vault or cuff scar following hysterectomy) is defined as POP

Female pelvic floor tissues are exposed to complex biomechanical environments including pregnancy, childbirth and other alterations in abdominal pressure. Coordination of ligaments, fascia and muscle support pelvic organs. Previous studies have demonstrated that POP is a disease based on the progressive decline and abnormalities of the biomechanical properties of pelvic support tissues $(4,5)$.

The etiology of POP is complex and multifactorial, and epidemiological studies have suggested that multiple pathologies contribute to full anatomical loss, involving vaginal parity and other obstetric risk factors $(6,7)$, in addition to advanced age, increased body mass index, smoking, constipation and vaginal hysterectomy (8-10). The mechanisms underlying pelvic floor support failure remain poorly understood; however, studies in humans and animals implicate defects in the extracellular matrix (ECM) or fibrous connective tissue causing reduced tissue strength and defective repair (11). It is hypothesized that alterations in the connective tissue and ECM of the pelvic organs may serve a role in the development of POP.

ECM composition, organization and compliance provide architectural and chemical cues that regulate tissue homeostasis. ECM produced by stromal fibroblasts serves a key role in POP. The ECM of the pelvic floor, termed the endopelvic fascia, is responsible for maintaining the position of organs 
adjacent to the vagina. The primary fibrillar components of the ECM, collagen and elastin, are hypothesized to contribute the most to its biomechanical properties. Their alterations are potentially involved in the physiopathology of POP (11). Collagen fibers are rigid and do not easily distort, whereas elastin fibers provide elasticity and recoil to the tissue. Elastic fibers are important for maintaining vaginal structural integrity against mechanical strain (12).

The ECM is degraded by a family of enzymes called the matrix metalloproteinases (MMPs). MMPs are Zn2+ dependent endopeptidases responsible for the ECM degradation of connective tissue matrix, including ligaments (13). Collectively, they are capable of degrading all types of ECM proteins (14). The gelatinase sub-family is composed of gelatinase A (MMP-2) and gelatinase B (MMP-9); the two are capable of metabolizing native and denatured collagen, gelatin, elastin, laminin, fibronectin and the basement membrane $(14,15)$.

Transforming growth factor- $\beta 1$ (TGF- $\beta 1$ ) has unique and widespread actions in the remodeling of the ECM and is critical for tissue integrity. TGF- $\beta 1$ is a multifunctional cytokine and dominant regulator of multiple ECM components and enzymes. Sustained elevations of TGF- $\beta 1$ have been associated with multiple pathological conditions, including pulmonary fibrosis, keloid formation, coronary artery restenosis and acute respiratory distress syndrome (16). Pascual et al (17) illustrated an increase in expression of TGF- $\beta 1$ in the fascia of inguinal hernias. The pathogenesis of abdominal hernias and POP may be similar, as the two conditions result in the loss of fascial support leading to a protrusion or herniation of organs. Previous studies have reported altered expression of TGF- $\beta 1$ in women with POP $(18,19)$. Our previous studies $(20,21)$ additionally demonstrated downregulation of TGF- $\beta 1$ in the pubocervical fascia tissue of patients with POP.

The aim of the present study was to investigate the expression of collagen and collagen metabolism-associated factors in human parametrial ligament fibroblasts (hPLFs) under mechanical strain and to examine potential alterations in collagen metabolism induced by mechanical strain in the pathogenesis of POP.

\section{Materials and methods}

Patients and primary culture of hPLFs. A total of 15 patients without POP or malignant tumors who underwent vaginal hysterectomy surgery at the Department of Obstetrics and Gynecology, Renmin Hospital of Wuhan University (Wuhan, China) were selected. Ethical approval was obtained from the Ethics Committee of Renmin Hospital of Wuhan University, following which all patients signed informed consent. The patients had no connective tissue diseases and oxidative stress-related diseases, including coronary heart disease, diabetes and hyperlipidemia. Patients with a history of estrogen application within the past three months were excluded from the study. Additionally, patients with endometriosis and ovarian, endocrine, tumor and estrogen-associated diseases, as confirmed by postoperative pathology, were removed from the study. Specimens were extracted from part of the uterosacral and cardinal ligaments in surgery. A collagenase digestion method was used for primary culture of hPLFs as described in our previous study (22).
Mechanical strain loading on hPLFs in vitro. Fibroblasts at generations 3-6 of exponential phase were selected and a four-point bending device (SXG4201; Miracle Technology Co., Ltd., Hsinchu, Taiwan) was used for mechanical loading. The specific methods used are described in our previous study (22). The fibroblasts were cultured on a specially-made plate (Miracle Technology Co., Ltd.) and stretched for mechanical strain loading. The parameters were set to a frequency of $0.5 \mathrm{~Hz}$ for $4 \mathrm{~h}$ and cells were subject to 1,333 or $5,333 \mu$, using $0 \mu$ strain as the control group.

Western blotting. Following mechanical strain loading at various degrees, the cells were lysed on ice for $30 \mathrm{~min}$ in RIPA buffer (Beyotime Institute of Biotechnology, Haimen, China) containing $1 \mathrm{mM}$ PMSF (Sigma, USA) and then centrifuged at $12,000 \times \mathrm{g}$ at $4^{\circ} \mathrm{C}$ for $15 \mathrm{~min}$. The supernatant was collected and quantified using a Bincinchoninic Acid protein assay kit (Beyotime Institute of Biotechnology). For each group, a total of $40 \mu \mathrm{g}$ protein was separated by SDS-PAGE with a 5\% stacking and a $10 \%$ separating gel, and subsequently transferred onto a polyvinylidene difluoride membrane. The membrane was blocked in $5 \mathrm{~g} / 1$ non-fat milk for $1 \mathrm{~h}$ and washed with TBS, following which it was incubated with the appropriate monoclonal antibodies at $4^{\circ} \mathrm{C}$ overnight. Following washing with TBS with Tween-20 (TBST), the membrane was incubated with a horseradish peroxidase-conjugated anti-IgG secondary antibody at $37^{\circ} \mathrm{C}$ for $1 \mathrm{~h}$. Subsequently, the membrane was washed with TBST and target proteins were visualized using an Enhanced Chemiluminescence kit (32106; Thermo Fisher Scientific, Inc., Waltham, MA, USA). The following primary antibodies were used: Collagen type I $\alpha 1$ chain (COL1A1; 1:1,000; ab34710), collagen type III $\alpha 1$ chain (COL3A1; 1:5,000; ab7778), elastin (1:1,000; ab23747), MMP-2 (1:200; ab7033), MMP-9 (1:1,000; ab38898) and TGF- $\beta 1$ (1:500; ab64715), all purchased from Abcam, Cambridge, MA, USA. GAPDH (1:1,000; ab8245; Abcam) was used as an internal reference control.

Reverse transcription-quantitative polymerase chain reaction $(R T-q P C R)$. Gene expression of COL1A1, COL3A1, MMP-2, MMP-9, TGF- $\beta 1$ and GAPDH were evaluated by RT-qPCR. The primers used for amplification were purchased from Beijing SBS Genetech Co., Ltd. (Beijing, China). Total RNA was extracted using TRIzol ${ }^{\circledR}$ (Invitrogen; Thermo Fisher Scientific, Inc., Waltham, MA, USA). RNA was reverse transcribed to cDNA using a RevertAid First Strand cDNA Synthesis kit (catalog no. k1622; Thermo Fisher Scientific, Inc.). SYBR ${ }^{\circledR}$ Premix Ex Taq ${ }^{\mathrm{TM}}$ (Takara Bio, Inc., Otsu, Japan) was used to detect gene expression in an ABI 7500 Real-Time PCR system (Applied Biosystems; Thermo Fisher Scientific, Inc.) for in vitro $\mathrm{qPCR}$ which was performed as follows: $30 \mathrm{sec}$ at $95^{\circ} \mathrm{C}, 40$ cycles of $5 \mathrm{sec}$ at $95^{\circ} \mathrm{C}, 34 \mathrm{sec}$ at $60^{\circ} \mathrm{C}, 15 \mathrm{sec}$ at $95^{\circ} \mathrm{C}, 1 \mathrm{~min}$ at $60^{\circ} \mathrm{C}, 15 \mathrm{sec}$ at $95^{\circ} \mathrm{C}$ and $15 \mathrm{sec}$ at $60^{\circ} \mathrm{C}$. mRNA expression levels were calculated and normalized to the expression levels of GAPDH. The primers used in this study are presented in Table I. Normalized quantitation threshold $(\mathrm{Cq})$ values were used for comparison (23). Each sample was analyzed in triplicate to ensure accuracy. 

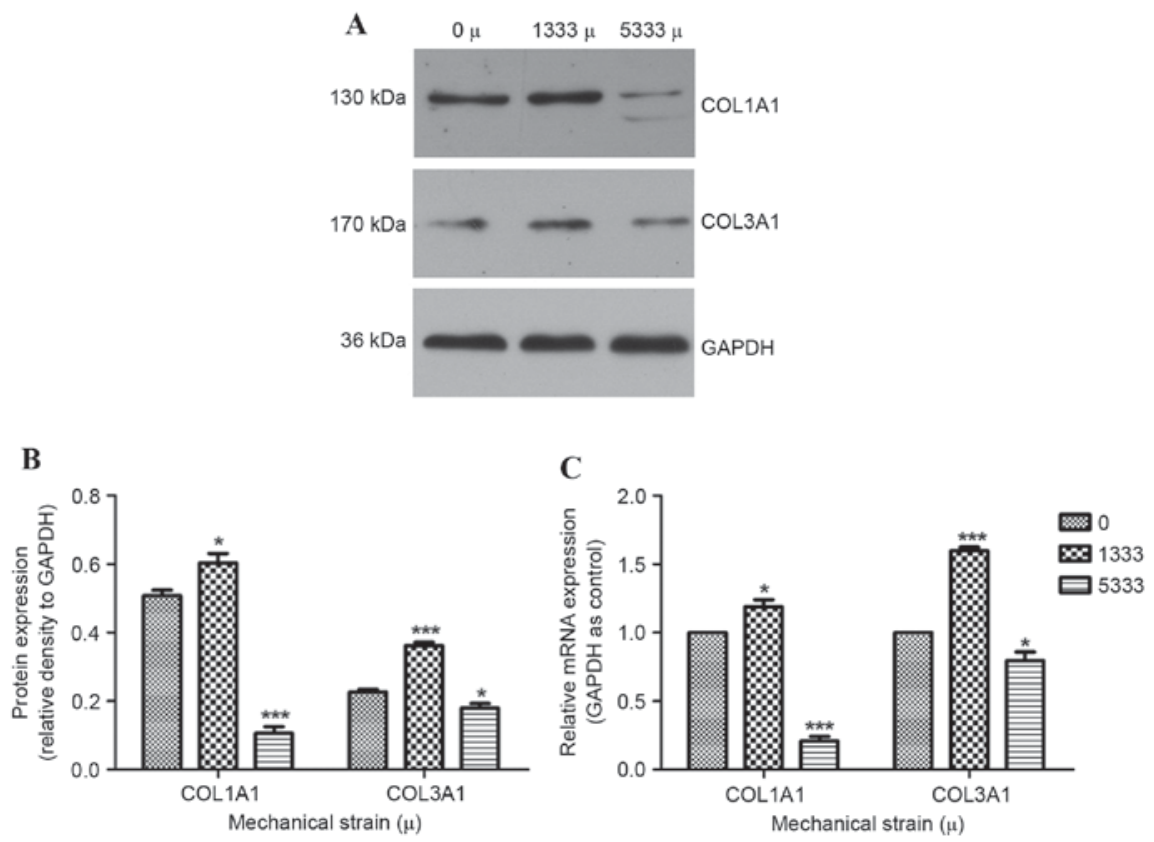

Figure 1. Protein and mRNA expression levels of COL1A1 and COL3A1 in human parametrial ligament fibroblasts following $0,1,333$ or 5,333 $\mu$ mechanical strain. (A) Representative western blot images and (B) quantification of protein expression levels of COL1A1 and COL3A1. GAPDH served as an internal control. (C) mRNA expression levels of COL1A1 and COL3A1 were detected by reverse transcription-quantitative polymerase chain reaction. Data are expressed as the mean \pm standard deviation. $\mathrm{P}<0.05$ vs. control $(0 \mu)$ group. ${ }^{* * *} \mathrm{P}<0.001$ vs. control $(0 \mu)$ group. COL1A1, collagen type I $\alpha 1$ chain; COL3A1, collagen type III $\alpha 1$ chain.

Table I. Primers used for reverse transcription-quantitative polymerase chain reaction.

\begin{tabular}{ll}
\hline Gene & \multicolumn{1}{c}{ Primer sequence (5'-3') } \\
\hline GAPDH & F: GCACCGTCAAGGCTGAGAAC \\
& R: TGGTGAAGACGCCAGTGGA \\
COL1A1 & F:CAAGACGAAGACATCCCACCAATC \\
& R: ACAGATCACGTCATCGCACAACA \\
COL3A1 & F: TCGCTCTGCTTCATCCCACTAT \\
& R: CTTCCAGACATCTCTATCCGCAT \\
Elastin & F: AAAGCAGCAGCAAAGTTCGG \\
& R: ACCTGGGACAACTGGAATCC \\
MMP-2 & F: AGTTCCATTCCGCTTCCAG \\
& R: CGGTCGTAGTCCTCAGTGGT \\
MMP-9 & F: GTCCACCCTTGTGCTCTTCC \\
& R: GACTCTCCACGCATCTCTGC \\
TGF- 31 & F: TATTGAGCACCTTGGGCACT \\
& R: ACCTCTCTGGGCTTGTTTCC
\end{tabular}

F, forward; R, reverse; COL1A1, collagen type III $\alpha 1$ chain; COL3A1, collagen type III $\alpha 1$ chain; MMP, matrix metalloproteinase; TGF, transforming growth factor.

Statistical analysis. Statistical analyses were performed with SPSS version 19.0 (IBM SPSS, Armonk, NY, USA). All data are expressed as the mean \pm standard deviation. ANOVA was performed to evaluate the differences between groups followed by Tukey post hoc test. $\mathrm{P}<0.05$ was considered to indicate a statistically significant difference.

\section{Results}

Expression levels of ECM components in hPLFs following mechanical strain. hPLFs were isolated and cultured from human uterosacral and cardinal ligaments and identified by positive staining of vimentin and negative staining of cytokeratin (22).

To examine whether the ECM serves an important role in the development of POP, western blotting and RT-qPCR were used to detect protein (Fig. 1A and B) and mRNA (Fig. 1C) expression levels of its components under various mechanical strains $(0,1,333$ or $5,333 \mu)$. As collagen is a primary component of the ECM, and COL1A1 and COL3A1 are the two principle components of pelvic connective tissue, their expression levels were detected. Under 1,333 $\mu$ mechanical strain, COL3A1 protein and mRNA expression levels were significantly increased compared with the control group $(\mathrm{P}<0.001)$. This trend was additionally observed in COL1A1 protein and mRNA expression levels $(\mathrm{P}<0.05)$. In contrast, decreased COL1A1 and COL3A1 protein and mRNA expression levels in response to 5,333 $\mu$ mechanical strain were observed, compared with the control group.

The effect of mechanical strain on elastin was examined, which is another primary fibrillar component of the ECM and provides elasticity and recoil to the ligament. As presented in Fig. 2, no significant differences in elastin protein (Fig. 2A and B) and mRNA (Fig. 2C) expression levels were observed between control cells and the $1,333 \mu$ mechanical strain group $(\mathrm{P}>0.05)$. On the other hand, the 5,333 $\mu$ mechanical strain group exhibited reduced protein (Fig. $2 \mathrm{~A}$ and $\mathrm{B}$ ) and mRNA (Fig. $2 \mathrm{C})$ expression levels $(\mathrm{P}<0.01$ and $\mathrm{P}<0.001$, respectively). Taken together, these results suggested that when hPLFs were 

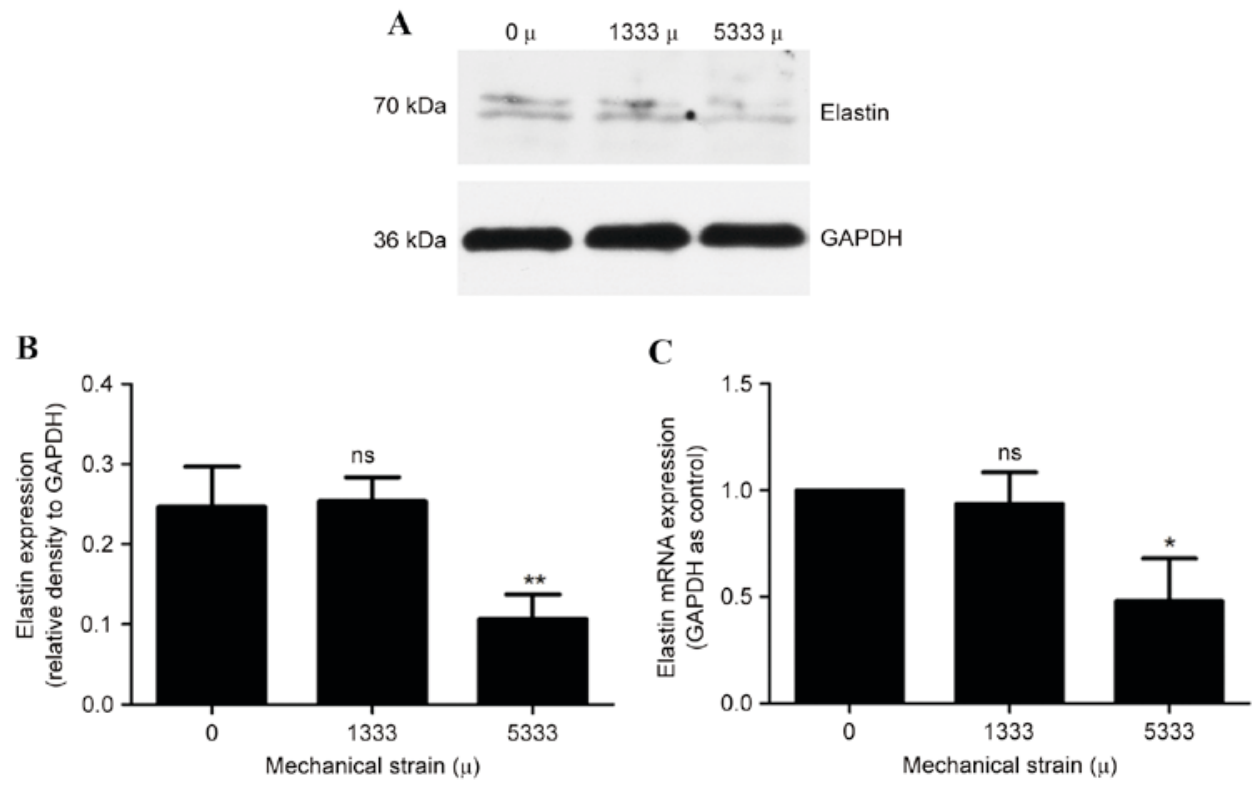

Figure 2. Protein and mRNA expression levels of elastin in human parametrial ligament fibroblasts following $0,1,333$ or 5,333 $\mu$ mechanical strain (A) Representative western blot images and (B) quantification of protein expression levels of elastin. GAPDH served as an internal control. (C) mRNA expression levels of elastin were detected by reverse transcription-quantitative polymerase chain reaction. Data are expressed as the mean \pm standard deviation. ${ }^{*} \mathrm{P}<0.05,{ }^{* * *} \mathrm{P}<0.001$ vs. control $(0 \mu)$ group. Ns, non-significant.
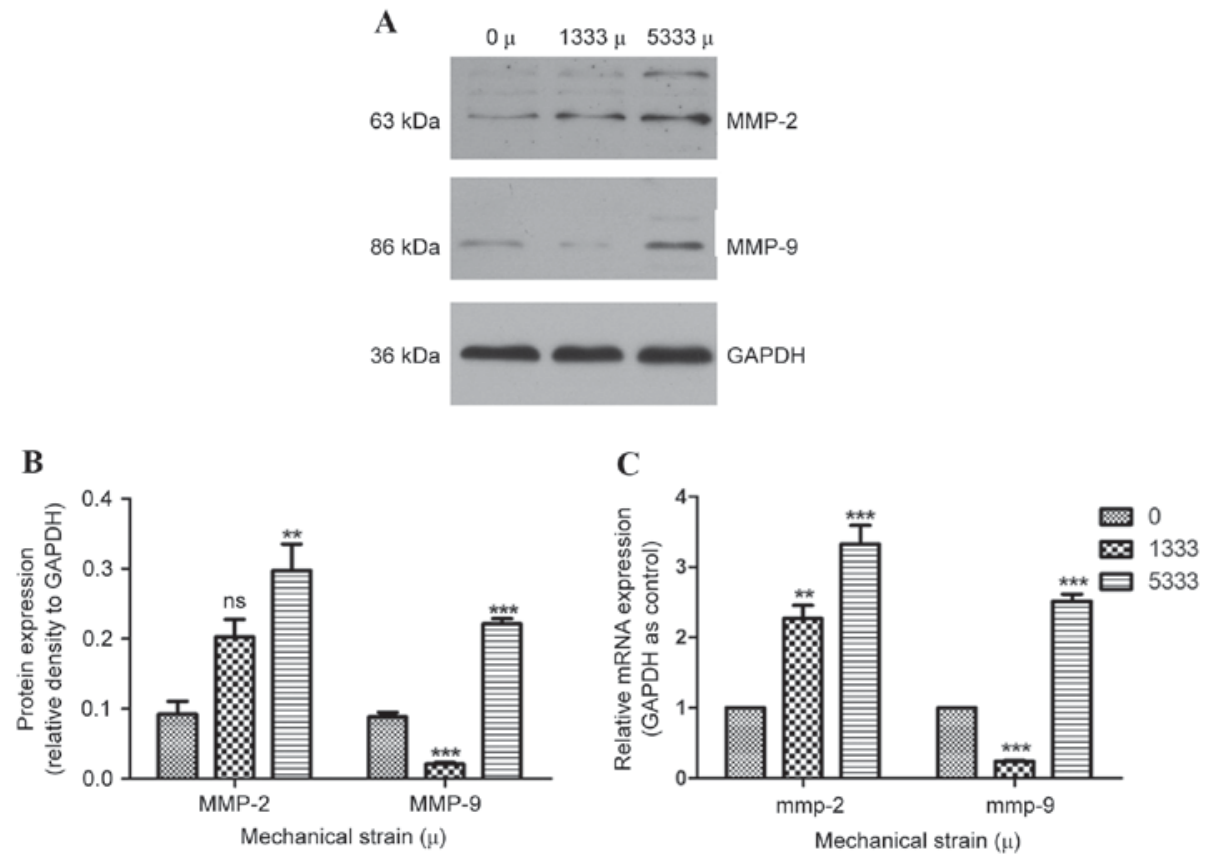

Figure 3. Protein and mRNA expression levels of MMP-2 and -9 in human parametrial ligament fibroblasts following 0, 1,333 or 5,333 $\mu$ mechanical strain (A) Representative western blot images and (B) quantification of protein expression levels of MMP-2 and -9. GAPDH served as an internal control. (C) mRNA expression levels of MMP-2 and -9 were detected by reverse transcription-quantitative polymerase chain reaction. Data are expressed as the mean \pm standard deviation. ${ }^{* *} \mathrm{P}<0.01,{ }^{* * *} \mathrm{P}<0.001$ vs. control $(0 \mu)$ group. MMP, matrix metalloproteinase; ns, non-significant.

subject to increased mechanical strain, expression levels of ECM components reduced.

Expression levels of MMP-2 and -9 in hPLFs following mechanical strain. MMP-2 and -9 are crucial enzymes for degrading the ECM; the production of MMP is associated with pelvic fibrosis. To demonstrate whether MMP is affected following the ECM alterations observed above, the expression levels of MMP-2 and -9 were assessed by western blotting and RT-qPCR. Compared with control cells, under 1,333 $\mu$ mechanical strain, no significant differences in MMP-2 protein expression levels were observed (Fig. 3A and B), whereas MMP-2 mRNA expression levels were significantly increased (P<0.01, Fig. 3C). Protein (Fig. 3A and B) and mRNA (Fig. 3C) expression levels of MMP-9 were reduced compared with the control group $(\mathrm{P}<0.001)$. When subject to $5,333 \mu$ mechanical 

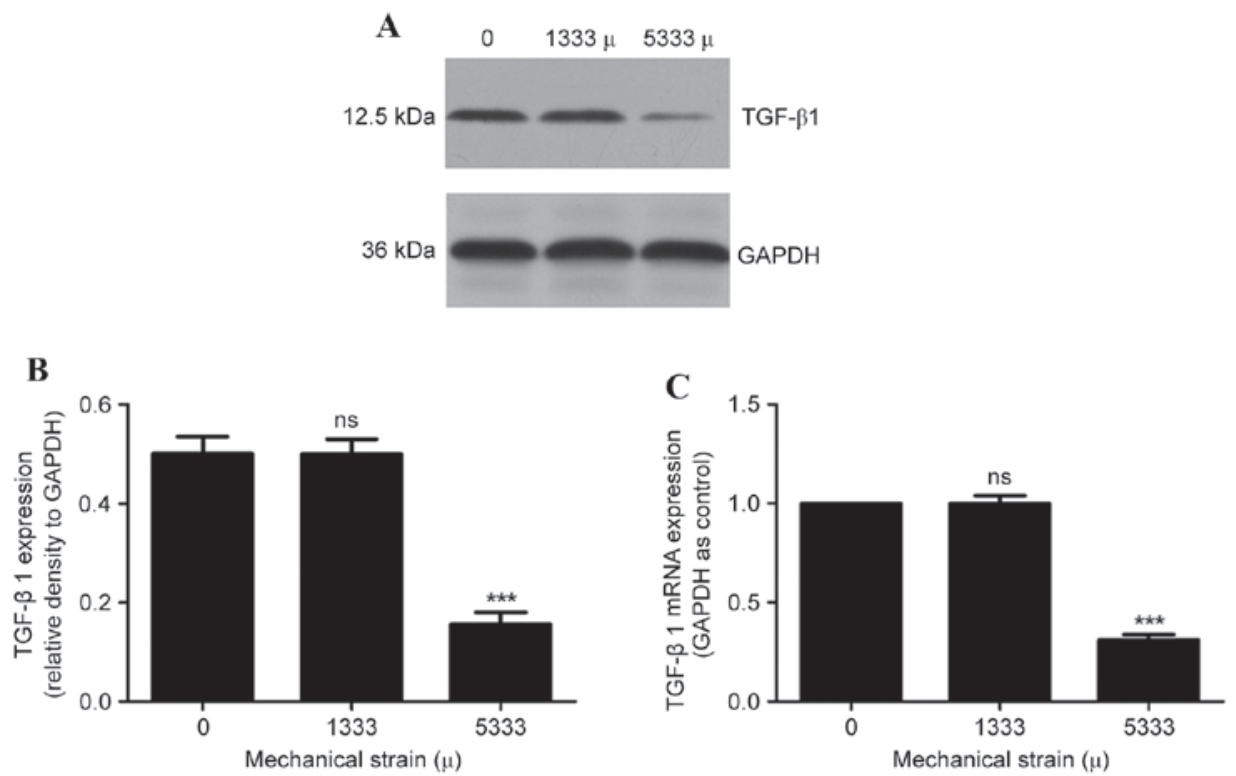

Figure 4. Protein and mRNA expression levels of TGF- $\beta 1$ in human parametrial ligament fibroblasts following $0,1,333$ or 5,333 $\mu$ mechanical strain (A) Representative western blot images and (B) quantification of protein expression levels of TGF-B1. GAPDH served as an internal control. (C) mRNA expression levels of TGF- $\beta 1$ were detected by reverse transcription-quantitative polymerase chain reaction. Data are expressed as the mean \pm standard deviation. ${ }^{* * *} \mathrm{P}<0.001$ vs. control $(0 \mu)$ group. Ns, non-significant; TGF- $\beta 1$, transforming growth factor $\beta 1$.

strain, MMP-2 and MMP-9 protein (Fig. 3A and B) and mRNA (Fig. 3C) expression levels were significantly increased compared with the control group $(\mathrm{P}<0.001)$.

Expression levels of TGF- $\beta 1$ in hPLFs following mechanical strain. TGF- $\beta 1$ is a multifunctional cytokine and dominant regulator of multiple ECM components and enzymes. No significant differences in TGF- $\beta 1$ protein (Fig. $4 \mathrm{~A}$ and B) and mRNA (Fig. 4C) expression levels were observed following $1,333 \mu$ mechanical strain, compared with compared with the control group $(\mathrm{P}>0.05)$. However, significantly decreased protein (Fig. 4A and B) and mRNA (Fig. 4C) expression levels of TGF- $\beta 1$ were observed following 5,333 $\mu$ mechanical strain $(\mathrm{P}<0.001)$.

\section{Discussion}

To the best of our knowledge, this study is the first evaluation of ECM metabolism using mechanical load in an hPLF model to investigate the potential pathogenesis of POP. These findings demonstrated that following mechanical strain, the expression of components of the ECM altered, particularly MMP- and -9 , and TGF- $\beta 1$, resulting in modified ECM metabolism. This indicated that the TGF- $\beta 1$ signaling pathway may be involved in the ECM disorder caused by mechanical strain.

POP is a common pelvic floor disorder (3), occurring when a loss of healthy attachment and support results in descent of the pelvic organs into the vaginal canal. DeLancey (24) reported three levels of connective tissue support of the vagina when defining pelvic floor anatomy. Level I refers to the apical portion of the posterior vaginal wall, which is suspended and supported primarily by the cardinal-uterosacral ligaments. If level I support is lost, apical POP follows. It has been reported that dysfunction of level I, comprised of the uterosacral and cardinal ligaments, is one of the key factors involved in
POP (25). Therefore, the present study selected hPLFs (derived from the uterosacral and cardinal ligaments) to examine.

Previous studies in pelvic tissues have demonstrated that alterations in the ECM lead to the development of POP. Collagen is the primary component of pelvic connective tissue, providing a scaffold for ECM assembly. Type I fibers are well-organized and present in uterosacral ligaments that provide DeLancey level I support of the cervix and vaginal apex (26). Type III fibers are more prominent in the loose areolar tissue surrounding the vagina and pelvic organs. Evaluation of the expression levels of COL1A1 and COL $3 \mathrm{~A} 1$ in women with and without POP has yielded varying results, with some studies demonstrating increased expression and others reporting decreased expression $(27,28)$. Numerous variables may contribute to this, including different tissue types being studied (for example uterosacral ligaments vs. the vaginal wall), harvesting and extraction methods, patient characteristics and the molecular makeup of the collagen. However, in the uterosacral and cardinal ligaments of patients with POP, numerous studies have demonstrated decreased COL1A1 and COL3A1 expression $(29,30)$. The present study on the expression levels of collagen in hPLFs following greater mechanical strain was consistent with a previous study in tissues (31), indicating that decreased collagen expression may serve an important role in the development of POP.

The results of the present study suggested that elastin expression levels additionally decreased following mechanical strain. Elastin fibers are key architectural elements of connective tissues that are subject to mechanical strain, providing extensibility and recoil ability to elastin tissues (32). Decrease in elastin content may cause alterations in the properties of the ligament, resulting in increased rigidity and decreased resistance to mechanical force. These findings supported the importance of collagen and elastin homeostasis in the development of POP. 
MMP-2 and -9 expression levels were examined in hPLFs following mechanical strain because of their important role in remodeling of the ECM.MMP-2 contains three repeats of a type II fibronectin domain in the catalytic domain, which binds to and may degrade gelatin, collagens and laminin (33). MMP-9 is a gelatinase whose primary function is the breakdown of basement membranes. Studies on the role of MMPs have resulted in divergent findings $(34,35)$. Dviri et al (36) demonstrated an increase in MMP-1 and -9 expression in uterosacral ligaments in women with POP. Conversely, Phillips et al (37) reported no difference in expression of MMP-9 in uterosacral ligaments in women with POP compared with controls. The present study demonstrated significantly increased mRNA and protein expression levels of MMP-2 and MMP-9 in hPLFs following $5,333 \mu$ mechanical strain, and decreased expression levels of the ECM components elastin, COL1A1 and COL3A1, which indicated that increased expression of MMP-2 and MMP-9 may lead to degradation of the ECM in POP development.

Studies examining the role of TGF- $\beta 1$ in POP have demonstrated contradictory results; Meijerink et al (19) reported a positive correlation between TGF- $\beta 1$ expression and POP, whereas Qi et al (20) observed a negative correlation. The present study demonstrated that mRNA and protein expression levels of TGF- $\beta 1$ were reduced following stronger mechanical strain $(5,333 \mu)$. The TGF- $\beta 1 /$ mothers against decapentaplegic homolog 3 (Smad3) signaling pathway is currently viewed as an important regulator of fibrosis and degenerative fibrotic diseases. Combined with these results, it was hypothesized that the TGF- $\beta 1 /$ Smad3 signaling pathway may serve a critical role in the development of POP, which requires further confirmation in future studies.

The present study additionally demonstrated that hPLFs subject to weak mechanical strain exhibited slightly altered ECM metabolism, resulting in an increase in synthesis of certain components of the ECM and a decrease in degradation. It is understood that clinical pelvic floor muscle training and other methods may contribute to pelvic recovery postpartum and may be used to treat pelvic floor dysfunction without symptoms (38). Therefore, moderate mechanical strain may be conducive to the maintenance and restoration of healthy pelvic tissue structure and functions. However, stronger mechanical strain may damage the pelvic floor tissue and POP develops. These results indicated that a weaker mechanical strain may benefit ECM metabolism, whereas a stronger mechanical strain may be harmful.

In conclusion, the present study demonstrated that upregulation of MMP expression and downregulation of the TGF- $\beta 1$ signaling pathway induced by stronger mechanical strain reduced ECM synthesis and increased ECM degradation. These in vitro results indicated that these factors may additionally be involved in the process of POP in vivo. This may supply a new target and strategy for the understanding of the etiology and treatment of POP.

\section{Acknowledgements}

The present study was supported by the National Natural Science Foundation of China (grant no. 81270684) and the Foundation of Collaborative and Innovation Projects of Wuhan University School of Medicine (grant no. 523-266078).

\section{References}

1. Wu JM, Matthews CA, Conover MM,Pate V and Jonsson Funk M: Lifetime risk of stress urinary incontinence or pelvic organ prolapse surgery. Obstet Gynecol 123: 1201-1206, 2014.

2. Buchsbaum GM, Duecy EE, Kerr LA, Huang LS, Perevich M and Guzick DS: Pelvic organ prolapse in nulliparous women and their parous sisters. Obstet Gynecol 108: 1388-1393, 2006.

3. Jelovsek JE, Maher C and Barber MD: Pelvic organ prolapse. Lancet 369: 1027-1038, 2007.

4. Dviri M, Leron E, Dreiher J, Mazor M and Shaco-Levy R: Increased matrix metalloproteinases-1, -9 in the uterosacral ligaments and vaginal tissue from women with pelvic organ prolapse. Eur J Obstet Gynecol Reprod Biol 156: 113-117, 2011.

5. Ewies AA, Al-Azzawi F and Thompson J: Changes in extracellular matrix proteins in the cardinal ligaments of post-menopausal women with or without prolapse: A computerized immunohistomorphometric analysis. Hum Reprod 18: 2189-2195, 2003.

6. Sandberg LB, Weissman N and Gray WR: Structural features of tropoelastin related to the sites of cross-links in aortic elastin. Biochemistry 10: 52-56, 1971.

7. Ramirez F, Sakai LY, Dietz HC and Rifkin DB: Fibrillin microfibrils: Multipurpose extracellular networks in organismal physiology. Physiol Genomics 19: 151-154, 2004.

8. Sakai LY, Keene DR and Engvall E: Fibrillin, a new 350-kD glycoprotein, is a component of extracellular microfibrils. J Cell Biol 103: 2499-2509, 1986.

9. Zhang H, Apfelroth SD, Hu W, Davis EC, Sanguineti C, Bonadio J, Mecham RP and Ramirez F: Structure and expression of fibrillin-2, a novel microfibrillar component preferentially located in elastic matrices. J Cell Biol 124: 855-863, 1994.

10. Carley ME and Schaffer J: Urinary incontinence and pelvic organ prolapse in women with Marfan or Ehlers Danlos syndrome. Am J Obstet Gynecol 182: 1021-1023, 2000.

11. Kerkhof MH, Hendriks L and Brölmann HA: Changes in connective tissue in patients with pelvic organ prolapse-a review of the current literature. Int Urogynecol J Pelvic Floor Dysfunct 20: 461-474, 2009.

12. Chen B, Wen Y and Polan ML: Elastolytic activity in women with stress urinary incontinence and pelvic organ prolapse. Neurourol Urodyn 23: 119-126, 2004.

13. Visse R and Nagase H: Matrix metalloproteinases and tissue inhibitors of metalloproteinases: Structure, function, and biochemistry. Circ Res 92: 827-839, 2003.

14. Lisboa RA, Lisboa FA, de Castro Santos G, Andrade MV and Cunha-Melo J: Matrix metalloproteinase 2 activity decreases in human periodontal ligament fibroblast cultures submitted to simulated orthodontic force. In Vitro Cell Dev Biol Anim 45: 614-621, 2009.

15. Yao PM, Buhler JM, d'Ortho MP, Lebargy F, Delclaux C, Harf A and Lafuma C: Expression of matrix metalloproteinase gelatinases $\mathrm{A}$ and $\mathrm{B}$ by cultured epithelial cells from human bronchial explants. J Biol Chem 271: 15580-15589, 1996.

16. Wilson MS and Wynn TA: Pulmonary fibrosis: Pathogenesis, etiology and regulation. Mucosal Immunol 2: 103-121, 2009.

17. Pascual G, Corrales C, Gómez-Gil V, Buján J and Bellón JM: TGF-betal overexpression in the transversalis fascia of patients with direct inguinal hernia. Eur J Clin Invest 37: 516-521, 2007.

18. Wen Y, Polan ML and Chen B: Do extracellular matrix protein expressions change with cyclic reproductive hormones in pelvic connective tissue from women with stress urinary incontinence? Hum Reprod 21: 1266-1273, 2006.

19. Meijerink AM, van Rijssel RH and van der Linden PJ: Tissue composition of the vaginal wall in women with pelvic organ prolapse. Gynecol Obstet Invest 75: 21-27, 2013.

20. Qi XY, Hong L, Guo FQ, Fu Q, Chen L and Li BS: Expression of transforming growth factor-beta 1 and connective tissue growth factor in women with pelvic organ prolapse. Saudi Med J 32: 474-478, 2011.

21. Li BS, Hong L, Min J, Wu DB, Hu M and Guo WJ: The expression of glutathione peroxidase-1 and the anabolism of collagen regulation pathway transforming growth factor-betal-connective tissue growth factor in women with uterine prolapse and the clinic significance. Clin Exp Obstet Gynecol 40: 586-690, 2013.

22. Hong S, Li H, Wu D, Li B, Liu C, Guo W, Min J, Hu M, Zhao Y and Yang Q: Oxidative damage to human parametrial ligament fibroblasts induced by mechanical stress. Mol Med Rep 12: 5342-5348, 2015 . 
23. Livak KJ and Schmittgen TD: Analysis of relative gene expression data using real-time quantitative PCR and the 2(-Delta Delta C(T)) method. Methods 25: 402-408, 2001.

24. Delancey JOL: Standing anatomy of the pelvic floor. J Pelvic Surg 2, 1996.

25. Ramanah R, Berger MB, Chen L, Riethmuller D and Delancey JO: See it in 3D!: Researchers examined structural links between the cardinal and uterosacral ligaments. Am J Obstet Gynecol 207: 437.e1-e7, 2012.

26. DeLancey JO: Anatomic aspects of vaginal eversion after hysterectomy. Am J Obstet Gynecol 166: 1717-1728, 1992.

27. Mosier E, Lin VK and Zimmern P: Extracellular matrix expression of human prolapsed vaginal wall. Neurourol Urodyn 29 $582-586,2010$

28. Connell KA, Guess MK, Chen H, Andikyan V, Bercik R and Taylor HS: HOXA11 is critical for development and maintenance of uterosacral ligaments and deficient in pelvic prolapse. J Clin Invest 118: 1050-1055, 2008.

29. Sun ZJ, Zhu L, Lang JH, Wang Z and Liang S: Proteomic analysis of the uterosacral ligament in postmenopausal women with and without pelvic organ prolapse. Chin Med J (Engl) 128: 3191-3196, 2015

30. Gabriel B, Denschlag D, Göbel H, Fittkow C, Werner M, Gitsch G and Watermann D: Uterosacral ligament in postmenopausal women with or without pelvic organ prolapse. Int Urogynecol J Pelvic Floor Dysfunct 16: 475-479, 2005.

31. Liu C, Yang Q, Fang G, Li BS, Wu DB, Guo WJ, Hong SS and Hong L: Collagen metabolic disorder induced by oxidative stress in human uterosacral ligament-derived fibroblasts: A possible pathophysiological mechanism in pelvic organ prolapse. Mol Med Rep 13: 2999-3008, 2016.
32. Kielty CM, Sherratt MJ and Shuttleworth CA: Elastic fibres. J Cell Sci 115: 2817-2828, 2002.

33. Creemers LB, Jansen ID, Docherty AJ, Reynolds JJ, Beertsen W and Everts V: Gelatinase A (MMP-2) and cysteine proteinases are essential for the degradation of collagen in soft connective tissue. Matrix Biol 17: 35-46, 1998.

34. Gabriel B, Watermann D, Hancke K, Gitsch G, Werner M, Tempfer $\mathrm{C}$ and zur Hausen A: Increased expression of matrix metalloproteinase 2 in uterosacral ligaments is associated with pelvic organ prolapse. Int Urogynecol J Pelvic Floor Dysfunct 17: 478-482, 2006.

35. Liang CC, Huang HY, Tseng LH, Chang SD, Lo TS and Lee CL: Expression of matrix metalloproteinase- 2 and tissue inhibitors of metalloproteinase-1 (TIMP-1, TIMP-2 and TIMP-3) in women with uterine prolapse but without urinary incontinence. Eur J Obstet Gynecol Reprod Biol 153: 94-98, 2010.

36. Dviri M, Leron E, Dreiher J, Mazor M and Shaco-Levy R: Increased matrix metalloproteinases-1,-9 in the uterosacral ligaments and vaginal tissue from women with pelvic organ prolapse. Eur J Obstet Gynecol Reprod Biol 156: 113-117, 2011.

37. Phillips $\mathrm{CH}$, Anthony $\mathrm{F}$, Benyon $\mathrm{C}$ and Monga AK: Collagen metabolism in the uterosacral ligaments and vaginal skin of women with uterine prolapse. BJOG 113: 39-46, 2006.

38. Trowbridge ER and Fenner DE: Conservative management of pelvic organ prolapse. Clin Obstet Gynecol 48: 668-681, 2005. 